\title{
Aviation Equipment Fault Information Fusion Based on Ontology
}

\author{
Yuan Wang ${ }^{1}$, Qing Li ${ }^{1}$, Yong Sun ${ }^{2}$, Jinliang Chen ${ }^{1}$ \\ ${ }^{1}$ Department of Mechanical Engineering and Automation, Beijing University of Aeronautics and Astronautics, \\ Beijing 100191, China \\ ${ }^{2}$ Chengdu Aircraft Design \& Research Institute, Chengdu 610091, China \\ wangyuan302308@126.com, liqing@buaa.edu.cn,sbomber@163.com,jinl_chen@163.com
}

\begin{abstract}
The designing institution, manufacturer and end-user jointly participate in aircraft maintenance. Maintenance data from multiple sources results in syntax and semantic conflicts, which have restricted integration and sharing of information on aircraft faults. To solve this problem, a solution based on ontology is proposed. Global aircraft fault ontology gives a uniform and specific description for multi-source data, eliminating the semantic heterogeneity. Data integration based on ontology mapping eliminates the structural heterogeneity. Ontology reasoning and data fusion algorithm is proposed and successfully implemented to remove the ambiguity and inconsistence from information on aircraft fault.

Index Terms - Ontology Mapping, Data Integration, Ontology Reasoning, Data Fusion, Aircraft Fault
\end{abstract}

\section{Introduction}

In China, multi-agent maintenance and support model is established for aviation equipment. Each agent (designing institution, manufacturer etc.) has developed information systems to meet their own needs. They don't have a uniform description for data definition and data structure, leading to semantic and syntax heterogeneity[1], which makes it difficult for data statistics, information sharing, quality analyses and quality controlling.

To make full use of aviation equipment fault information and eliminate the semantic and syntax heterogeneity, this paper adopts information fusion method based on ontology[2] to establish fault information sharing mechanism. Ontology has a clear conceptual hierarchy and explicit semantic description[3]. Therefore, we use ontology as public model. Taking advantage of semantic description, querying and logical reasoning, ontology is adopted to remove the information redundancy and heterogeneity such as data inconsistency and sematic ambiguity.

\section{Data Fusion Framework}

Figure 1 shows the aircraft fault information fusion frame based on ontology. First, a global aircraft fault ontology is built to avoid semantic heterogeneity. Second, ontology mapping is applied to integrate multiple sources into an ontology library, eliminating syntax heterogeneity. Finally, Jess and SWRL rules are adopted to reason and fuse the redundant and incomplete fault information.

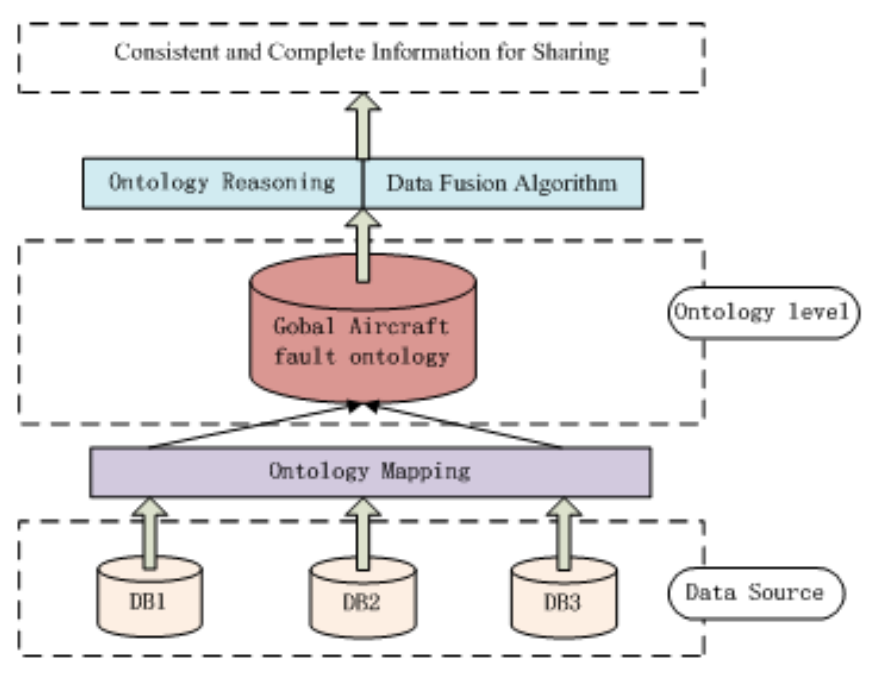

Figure 1 Information Fusion Frame

\section{Ontology Modeling}

By analyzing the data of aircraft fault in service phase and consulting experts, we divide the aircraft fault knowledge[4] into three categories, including product knowledge, fault case knowledge and fault diagnosis knowledge. Each category contains fault concepts which are closely linked, as shown below.

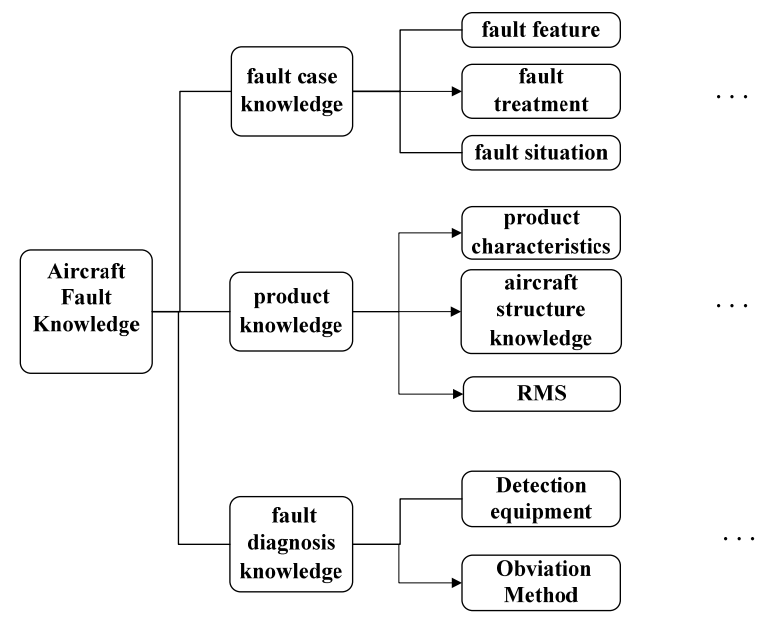

Figure 2 Aircraft Fault Knowledge 
Descriptions are as follows:

Product knowledge is very extensive, we mainly research the fault-related product knowledge, including product characteristics, aircraft structure knowledge, RMS(Reliability, Maintainability, Supportability)[5], etc. A Fault case is a complete record of fault. A great deal of fault cases are collected during the aircraft maintenance. The cases contain not only the environmental information but also the professional knowledge of related fields. This paper includes such fault knowledge as fault feature, fault treatment, fault situation, support resources, etc. Fault diagnosis[6] is the process of fault detection and isolation and fault diagnosis knowledge is also the basis of fault handling.

The core concept of the aircraft fault ontology is extracted. It includes fault components, fault mode, fault cause, fault consequence and fault handling. Figure 3 illustrates the frame of the concepts above.

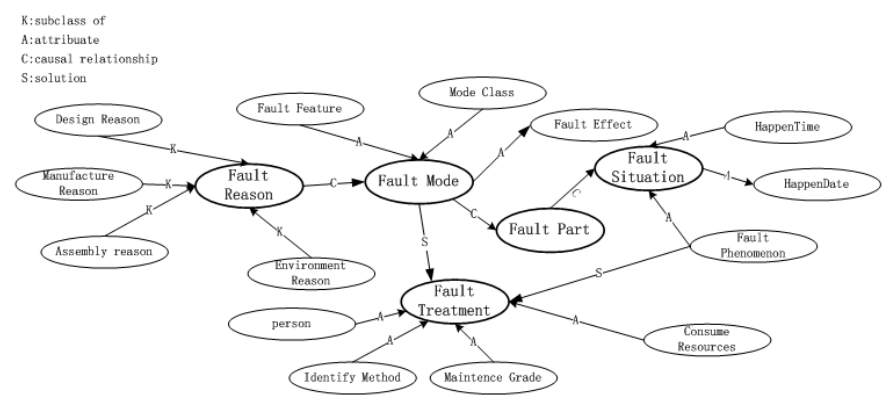

Figure 3 Aircraft Fault Ontology Frame

It is essential to encode the ontology with formal methods so that the computer can interpret the words. Protégé, which is a open-source ontology coding tools developed by Stanford University, is used in this paper with an ontology language OWL[7]. It is recommended by $\mathrm{W} 3 \mathrm{C}$.

\section{Data Integration Based on Ontology Mapping}

Different data structure designers have different understanding of the same object[8], leading to data heterogeneity. Similarly, as the designing institution, manufacturer and end user focus on different aspects, they describe aircraft fault in different ways. As a result, information systems developed by them have semantic and syntax conflicts including mode layer conflict and data layer conflict.

The mode layer conflict mainly appears as attribute conflict[9] and attribute set conflict. Attribute conflict refers to that different data sources using different definitions for the same attributes. For example, a customer uses "problem" to define fault phenomenon while the designing institution uses "fault description". Attribute set conflict refers to that the same entity in different system has different attribute sets. For example, the entity "fault handling" has an attribute set (fault treatment class, maintenance grade, fault detection method and handler). However, when it comes to another system, it has another attribute set (fault treatment class, maintenance grade, handlers).
Data conflicts are mainly conflicts on data accuracy, format, integrity constraints and other aspects. It mainly appears as data type conflict, data format conflict, data unit conflict, data accuracy conflict, etc.

The aircraft fault ontology provides consistent and uniform ontology semantic for heterogeneous data sources[1], and as a result the attribute conflict is removed. In the aircraft fault ontology modeling process, the tables, attributes, and relationships of data sources are either merged or copied, to solve the attribute set conflicts. Some of the semantic mapping relationship is shown in the following table.

Table 1

\begin{tabular}{|l|l|l|}
\hline Global ontology & Data source 1 & Data source 2 \\
\hline Fault component NO. & Product number & Fault component NO. \\
Fault components' name & Product name & Name \\
Fault phenomenon & Problems & Fault description \\
Fault occurring time & Occurring time & Occurring time \\
Model and type NO & Model and type & Type of fault part \\
$\ldots \ldots$ & $\ldots \ldots$ & $\ldots . . .$. \\
\hline
\end{tabular}

Entity of relational database corresponds to ontology class, while attribute corresponds to ontology data property and record in the relational database corresponds to ontology instance[10]. The mapping between relational database and ontology is the basis of information fusion.

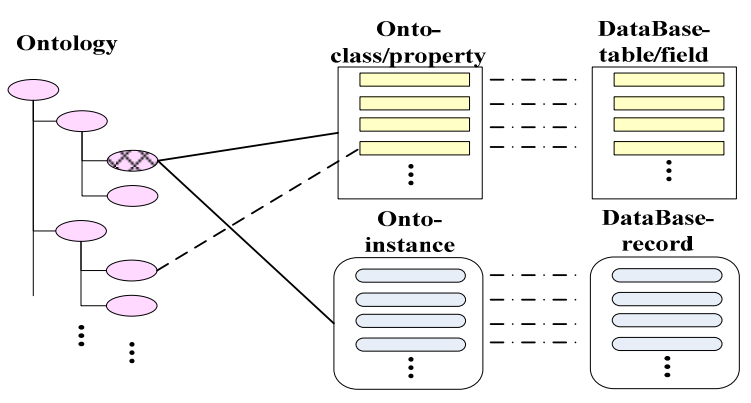

Fault information systems of an aircraft designing institution and an aircraft manufacturer are studied. Based on ontology mapping[11] result and Java coding with the OWL API, information in these two databases are imported to the aircraft fault ontology, the transforming message can be shown.

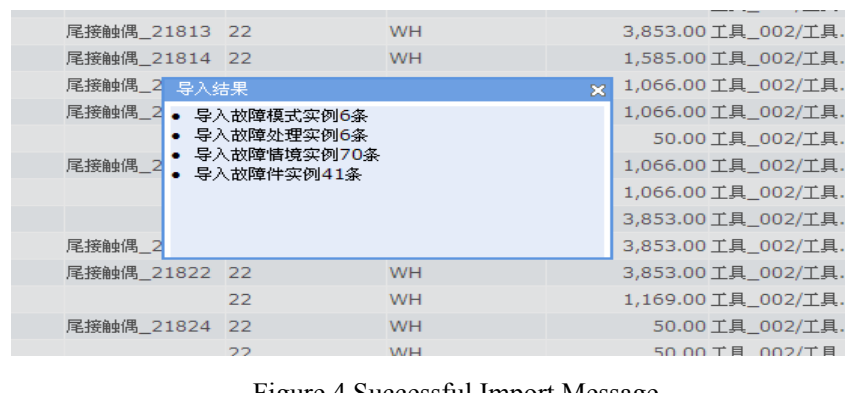

\section{Data Fusion Based on Ontology Reasoning}

Data integration[12] based on ontology- mapping solved the shcema and semantic heterogeneity of data from multiple 
sources, but massive data that is integrated into the ontology can inevitably be redundant. The same fault information might be recorded by the aircraft designing institution and manufacturer at the same time. In addition, neither of them has a complete description of fault information. Therefore, redundant and incomplete fault information should be fused into consistent and complete fault information after data integration.

The same fault instance is defined as the following: has the same property set (plane NO, plane type, faultcomponents' name, model and type NO, fault description, fault mode, fault occurring time, occurring date, fault treatment ) and has different fault component ID. In this paper, SWRL (Semantic Web Rule Language) rules and ontology reasoning machine[13] are used to recognize the same fault information, the decision rule in SWRL is:

Same_FaultCase: $\quad$ Fault_Part(?x) $\wedge$ Fault_Part $(? \mathrm{y}) \wedge$ HasSimilar_FaultCase $(? \mathrm{x}, ? \mathrm{y}) \wedge$ HasFault_Situation $(? \mathrm{x}, ? \mathrm{z}) \wedge$ Happen Time(?z, ?a) $\wedge$ Happen Date(?z, ?b) $\wedge$ HasFault Treament(?x, ?c) $\wedge$ Name(?c, ?d) $\wedge$ HasFault_Situation (?y, ?f) $\wedge$ Happen Time (?f, ?g) $\wedge$ Happen Date (?f, ?h) $\wedge$ HasFault_Treament (?y, ?i) $\wedge$ Name(?i, ?j) $\wedge$ swrlb: equal(?a, ?g) $\wedge$ swrlb: equal(?b, ?h) $\wedge$ swrlb: equal(?d, ?j) $\rightarrow$ HasSame_FaultCase $(? \mathrm{x}, ? \mathrm{y}) \wedge$ HasSame_FaultCase $(? \mathrm{y}, ? \mathrm{x})$

With Jess as ontology reasoning engine[14], combined with the SWRL rules and the fault ontology, all the redundant fault information are removed. Finally the data fusion algorithm is adopted to fuse these redundant fault information, algorithm as shown in the figure below:

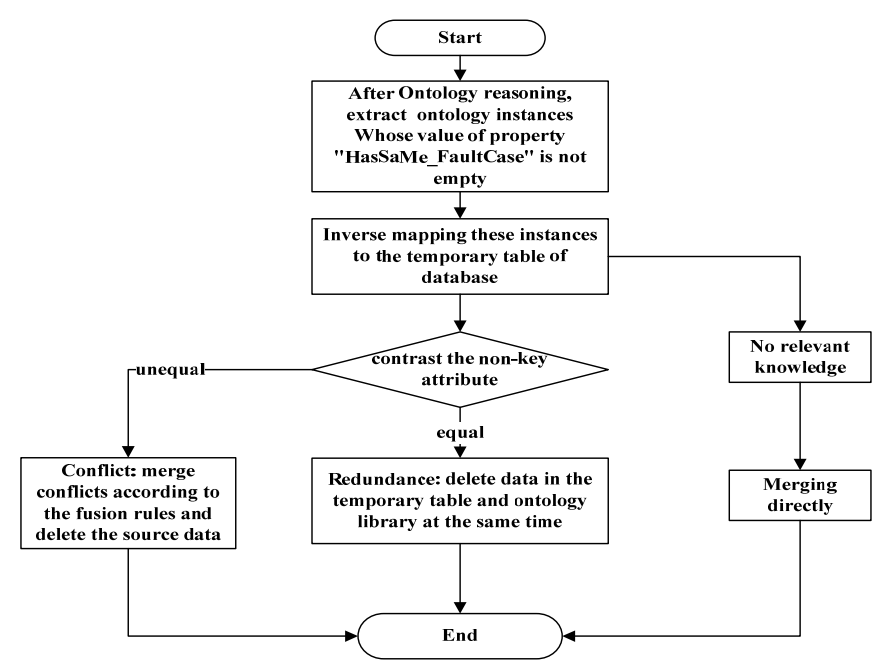

Above all, heterogeneous information fusion prototype system is developed, with information fusion module as shown in the figure below:

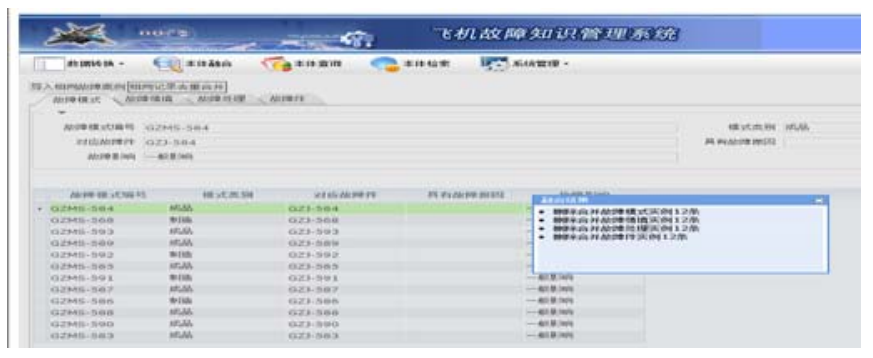

\section{Conclusion}

Ontology representation, information integration and ontology reasoning are applied to the aircraft fault information management. On this basis, information fusion prototype system is developed. It provides a uniform description for fault information, and transforms the redundant and incomplete fault information into consistent and complete information. The methods mentioned in this paper effectively promote aircraft fault information accumulation and sharing.

\section{References}

[1] Li Xiaohui, Wang Panqing, Wang Yinglong. Information integration research in equipment field based on otology. Computer Technology and Development. 2011(21).

[2] Xiao J. F. Song W. ontology - based Information Retrieval Model for the Semantic Web. IEEE. 2005.

[3] Wen Jingqian, Li Qing. Ontology-based semantic smiilarity computation formaintenance CBR. Journal of Beijing University of Aeronautics and Astronautics. 2009(02): 223-226.

[4] Zhou Yang. Ontology-based aircraft fault knowledge management research. Beijing: Beijing University of Aeronautics and Astronautics, 2011.

[5] Kong Fanke, Wang Yuquan. Reaserch on Equipment Quality Problem of Use Phase. Journal of Armored Fource Engineering Institute.2001.

[6] Wang Jianjun. Structure of Expert Knowledge Database of Aircraft Failures. Development \& Innovation of Machinery \& Electrical Products. 2012(01).

[7] Uschold M, Gruninger M. Ontologies: Principles, methods and applications. Knowledge engineering review. 1996, 11(2): 93-136.

[8] Li Yanxia. Information Integration Technology to Eliminate Semantics Heterogeneous. 2009.

[9] Wiesner A, Morbach J, Marquardt W. Information integration in chemical process engineering based on semantic technologies. Computers \& Chemical Engineering. 2011, 35(4): 692-708.

[10] Zhang Xiaoming, Hu Changjun. Survey on Mapping from Relational Database to Ontology. Journal of Chinese Computer Systems. 2009.

[11] Zhu Fanwei. Ontology-based heterogeneous data integration key technologies. Zhejiang University, 2008.

[12] Lou Shuangshuang. Ontology-based information fusion service mechanism research and practice. Beijing University of Posts and Telecommunications, 2008.

[13] Guo Wenying. Semantic Discovery and Ontology Mapping and Integration based on SWRL Reasoning. Zhejiang University, 2006.

[14] lin Xiao,Li Shaowen. Study on Ontology-based Reasoning System for Rice Pest Diagnosis. Agriculture Network Information. 2011(01): 17-20. 